\title{
Sarcoidosis and Obstructive Sleep Apnea: Yet another Overlap Syndrome?
}

\section{Andre Holmes, Charlie Strange and Chitra Lal*}

Division of Pulmonary, Critical Care, Allergy and Sleep Medicine, Medical University of South Carolina, 96 Jonathan Lucas Street, CSB 812, MSC 630, Charleston, SC 29425, USA

Sarcoidosis is a multisystem granulomatous disorder of unknown etiology that can affect any organ system in the body. Commonly, it involves the lungs and can present in various stages. Sarcoidosis may involve the upper respiratory tract in $2 \%-6 \%$ of patients [1].

Obstructive Sleep Apnea Syndrome (OSAS) is a common disorder which is often under diagnosed. Since OSAS is a major health problem with significant long term sequelae, there is a major interest in the prevention, early diagnosis, and adequate treatment of OSAS. Since the pathogenesis of OSAS is incompletely defined, rare diseases such as sarcoidosis may provide insight into OSAS causation, if there is a link between untreated sarcoidosis and OSAS development. Potential pathogenic mechanisms would include upper airway involvement by granulomatous inflammation, hypotonicity of the upper airway due to sarcoid myopathy or neuropathy or another unrecognized mechanism.

Sarcoid lesions in the upper airway can consist oflarge granulomatous lesions and nodules of the epiglottis and other supraglottic structures. Adhesions due to chronic inflammation and scarring may occur [2]. An epidemiologic study of 736 sarcoid patients in the United States revealed involvement of the ear, nose and throat in $2.3 \%$ of patients [3]. Visualization of the oropharyngeal cavity with a laryngoscope may reveal epiglottic thickening and granularity, granulomatous masses, and infiltrative sarcoid nodules of the epiglottis, aryepiglottic folds, and false cords [4]. Occasionally, laryngeal sarcoid can lead to progressive lifethreatening airway obstruction. The incidence of laryngeal sarcoidosis is estimated at $1.2 \%$ [5]. There are case reports of the occurrence of OSAS due to laryngeal involvement with sarcoidosis [6].

OSAS has been reported to occur with a higher frequency in sarcoid patients, with one study reporting a prevalence of $17 \%$ as opposed to 3\% in the non-sarcoid control group [7]. The exact reasons for a higher prevalence of OSAS in sarcoidosis are not fully understood; however, any of the mechanisms described above could predispose to the development of OSAS or worsen pre-existing OSAS. Other theories suggest that obesity resulting from excessive corticosteroid use increase neck circumference and narrow the upper airway. Potential associations with lupus pernio and neurosarcoidosis have been correlated with a higher prevalence of OSAS [7].

Although the impact of OSAS in sarcoidosis patients remains to be defined, both diseases can cause fatigue $[8,9]$. Since OSAS related fatigue is treated with continuous positive airway pressure (CPAP) and sarcoid related fatigue may require immunosuppressive therapy, it is important to differentiate these two conditions. The overlap between sarcoidosis and OSAS could also have significant adverse effects on lung physiology. Since the nocturnal hypoxemia resulting from this overlap syndrome might be worse than hypoxemia from either disease alone, the impact on cardiovascular co-morbidities could be exponentially higher. These detrimental effects of sleep-related hypoventilation and hypoxemia may include pulmonary hypertension, cerebrovascular disease with associated neurocognitive deficits and coronary artery disease.

Management of obesity is an important aspect of pulmonary practice. As a specialty that uses a disproportionate share of corticosteroids, we have a duty to be sensitive to the possibility of OSAS development. While future studies will be required to define if an overlap syndrome between sarcoidosis and OSAS exists independent of corticosteroid induced obesity, the possibility exists that patients will be harmed if OSAS is left untreated. Future studies should target the possible pathogenetic mechanisms that underlie the relationship between OSAS and sarcoidosis. A better understanding of this overlap syndrome might also help in the evolution of new treatment modalities and therapeutic interventions for both disorders.

\section{References}

1. Polychronopoulos VS, Prakash UB (2009) Airway involvement in sarcoidosis. Chest 136: 1371-1380

2. James DG, Barter S, Jash D, MacKinnon DM, Carstairs LS (1982) Sarcoidosis of the upper respiratory tract (SURT). J laryngol otol 96: 711-718.

3. Baughman RP, Teirstein AS, Judson MA, Rossman MD, Yeager $\mathrm{H}$ Jr, et al. (2001) Clinical characteristics of patients in a case control study of sarcoidosis Am J Respir Crit Care Med 164: 1885-1889.

4. Carasso B (1974) Sarcoidosis of the larynx causing airway obstruction. Chest 65: 693-695.

5. Weiss JA (1960) Sarcoidosis in otolaryngology. Report of eleven cases. Evaluation of blind biopsy as a diagnostic aid. Laryngoscope 70: 1351-1398.

6. Shah RN, Mills PR, George PJ, Wedzicha JA (1998) Upper airways sarcoidosis presenting as obstructive sleep apnoea. Thorax 53: 232-233.

7. Turner GA, Lower EE, Corser BC, Gunther KL, Baughman RP (1997) Sleep apnea in sarcoidosis. Sarcoidosis Vasc Diffuse Lung Dis 14: 61-64.

8. Hinz A, Fleischer M, Brahler E, Wirtz H, Bosse-Henck A (2011) Fatigue in patients with sarcoidosis, compared with the general population. Gen Hosp Psychiatry 33: 462-468.

9. Lurie A (2011) Obstructive sleep apnea in adults: epidemiology, clinical presentation, and treatment options. Adv Cardiol 46: 1-42.
*Corresponding author: Chitra Lal, MD, D-ABSM, FCCP, Assistant Professor of Medicine, Medical Division of Pulmonary, Critical Care, Allergy and Sleep Medicine, Medical University of South Carolina, 96 Jonathan Lucas Street CSB 812, MSC 630, Charleston, SC 29425, USA, Tel: (843)792-7776; E-mail: lalch@musc.edu, chitra_lal@hotmail.com

Received March 20, 2012; Accepted March 22, 2012; Published March 24, 2012

Citation: Holmes A, Strange C, Lal C (2012) Sarcoidosis and Obstructive Sleep Apnea: Yet another Overlap Syndrome?. J Pulmonar Respirat Med 2:e113. doi:10.4172/2161-105X.1000e113

Copyright: @ 2012 Holmes A, et al. This is an open-access article distributed under the terms of the Creative Commons Attribution License, which permits unrestricted use, distribution, and reproduction in any medium, provided the original author and source are credited. 